\title{
Genetically modified mosquitos may be used in fight against Zika
}

\author{
Anne Gulland \\ London
}

The World Health Organization is considering introducing transgenic mosquitos as a way of tackling the Zika virus.

WHO's vector control advisory group is to be convened in the next few weeks to look at how to control the Aedes Aegypti mosquito, the main vector of the Zika virus. Besides looking at conventional vector control techniques, the group will consider the introduction of transgenic mosquitoes.

Pedro Alonso, director of WHO's global malaria programme, told a press conference that controlling A Aegypti was complex. These mosquitoes have adapted well to human environments, live in urban areas, and have a particular attraction to humans. They bite during the day, unlike most malaria transmitting mosquitoes, and their eggs are sticky so are difficult to eliminate. Their larvae can also survive for many months.

"They have a good capacity to survive in complex situations. The females tend to bite multiple times and may lay eggs in different places. This makes it a particularly effective vector to transmit a pathology," he said.

Alonso added that WHO had been considering the introduction of transgenic mosquitos for some time. He said, "These [transgenic] mosquitos can reproduce, but the larvae die early on and do not reach adulthood. They have shown promising results as a mechanism to reduce the population density of $A$ Aegypti, but we have not yet seen the full public health value," he said.

WHO has been looking at the OX513A mosquito, developed by the UK biotechnology firm Oxitec, which has already been introduced into Brazil in a bid to tackle dengue fever, also carried by A Aegypti. Genetically modified male mosquitos are released into the population to mate with wild female mosquitos. The male insects have been shown in laboratory tests to be successful at competing with wild male populations.

Concerns have been expressed that this genetically modified mosquito is responsible for the increase in the number of babies born with microcephaly in Brazil, but a statement on WHO's website says that there is no evidence that Zika virus disease or the increase in incidence of microcephaly is caused by genetically modified mosquitoes.
The statement continues, "WHO encourages affected countries and their partners to boost the use of current mosquito control interventions as the most immediate line of defence, and to judiciously test the new approaches that could be applied in future."

Other new techniques considered by WHO include the introduction of male mosquitoes carrying Wolbachia, a genus of bacteria that affects only invertebrates and that can shorten the lifespan of mosquitos and reduce the amount of viruses that mosquitos harbour and transmit to humans. Alonso said that that this technique had been under consideration for the past three years but had not yet been properly evaluated.

Another technique involves the release of male insects that have been sterilised with low doses of radiation. Alonso said this was a "potentially interesting concept" that WHO had not yet considered.

Between January 2007 and February this year 41 countries reported transmission of the Zika virus, with six countries or territories reporting an increase in the incidence of microcephaly in newborns or of Guillain-Barré syndrome. Brazil has seen the highest number of babies with microcephaly.

No definitive link between the virus and these two syndromes has yet been found, but Bruce Aylward, WHO's executive director for outbreaks and health emergencies, said that the organisation was working on the presumption of "guilty until proved innocent."

He said, "There have been a number of autopsies and investigations into microcephaly and children where they have found evidence of the virus in the body. This does not prove causation, but it's an increase in the accumulation of the evidence," he said.

For all The BMJs articles on the Zika epidemic go to bmj.co/zika.

Published by the BMJ Publishing Group Limited. For permission to use (where not already granted under a licence) please go to http://group.bmj.com/group/rights-licensing/ permissions 